\title{
A Phase II Study of Additional Four-Week Chemotherapy With Capecitabine During the Resting Periods After Six- Week Neoadjuvant Chemoradiotherapy in Patients With Locally Advanced Rectal Cancer
}

\author{
Kyung Ha Lee, Min Sang Song, Jun Boem Park, Jin Soo Kim, Dae Young Kang ${ }^{1}$, Ji Yeon Kim \\ Departments of Surgery and ${ }^{1}$ Pathology, Chungnam National University Hospital, Daejoen, Korea
}

Purpose: The aim of this study is to evaluate the efficacy and the safety of additional 4 -week chemotherapy with capecitabine during the resting periods after a 6-week neoadjuvant chemoradiotherapy (NCRT) in patients with locally advanced rectal cancer.

Methods: Radiotherapy was delivered to the whole pelvis at a total dose of 50.4 Gy for 6 weeks. Oral capecitabine was administered at a dose of $825 \mathrm{mg} / \mathrm{m}^{2}$ twice daily for 10 weeks. Surgery was performed 2-4 weeks following the completion of chemotherapy.

Results: Between January 2010 and September 2011, 44 patients were enrolled. Forty-three patients underwent surgery, and 41 patients completed the scheduled treatment. Pathologic complete remission (pCR) was noted in 9 patients (20.9\%). $\mathrm{T}$ down-staging and $\mathrm{N}$ down-staging were observed in 32 patients $(74.4 \%)$ and 33 patients $(76.7 \%)$, respectively. Grade 3 to 5 toxicity was noted in 5 patients $(11.4 \%)$. The pCR rate was similar with the pCR rates obtained after conventional NCRT at our institute and at other institutes.

Conclusion: This study showed that additional 4-week chemotherapy with capecitabine during the resting periods after 6-week NCRT was safe, but it was no more effective than conventional NCRT.

Keywords: Rectal neoplasms; Neoadjuvant therapy; Chemoradiotherapy; Capecitabine

\section{INTRODUCTION}

Neoadjuvant chemoradiotherapy (NCRT) has been used as standard treatment modality in patients with locally advanced rectal cancer (LARC) because of increased local control and less toxicity [1]. NCRT has enhanced down-staging of tumors and has reduced

Received: May 13, 2013 - Accepted: August 7, 2013

Correspondence to: Ji Yeon Kim, M.D.

Department of Surgery, Chungnam National University Hospital,

282 Munhwa-ro, Jung-gu, Daejeon 301-721, Korea

Tel: +82-42-280-7175, Fax: +82-42-257-8024

E-mail:jkim@cnu.ac.kr

(c) 2013 The Korean Society of Coloproctology

This is an open-access article distributed under the terms of the Creative Commons Attribution NonCommercial License (http://creativecommons.org/licenses/by-nc/3.0) which permits unrestricted non-

commercial use, distribution, and reproduction in any medium, provided the original work is properly cited. the local recurrence to below $10 \%$ [2-4]. However, the survival benefit of NCRT has not been demonstrated [1-3], and the rate of distant metastasis was still reported to be as high as $30 \%$ [4].

Patients with pathologic complete remission (pCR) after NCRT have shown excellent survival benefit, and several studies demonstrated that $\mathrm{pCR}$ was a significant prognostic factor for rectal cancer patients who received NCRT $[5,6]$. The pCR rate has been reported as $15-30 \%$ [7]. Numerous investigators have tried diverse chemotherapeutic regimens to increase the $\mathrm{pCR}$ rate, resulting in improved survival [7]. These efforts were based on the fact that addition of chemotherapy to neoadjuvant radiotherapy (RT) enhanced the radiation effect as a radiosensitizer $[3,8,9]$. However, the efficacy of NCRT as a tumoricidal agent in and of itself has remained unclear. There has been no single chemotherapeutic agent increasing the pCR significantly until now. If increasing the duration of chemotherapy improved the effect of NCRT, it might 
be a reasonable way to increase the $\mathrm{pCR}$ rate and improve survival.

Conventional NCRT has a resting period of 4-8 weeks after completion of treatment. However, Habr-Gama et al. [10] reported a $65 \%$ clinical or pCR rate after an additional 3 cycles of identical chemotherapy during the resting period following completion of 6-week 5-fluorouracil (5-FU)-based chemoradiotherapy (CRT). They suggested that additional chemotherapy might increase the pCR rate through both a radiosensitizing effect and a potential antitumor effect. Our institute has used capecitabine as a neoadjuvant chemotherapeutic agent. We inferred that additional chemotherapy during the resting period could enhance the $\mathrm{pCR}$ rate. The aim of the present study is to evaluate the safety and the efficacy of an additional 4-week chemotherapy with capecitabine after the conventional 6-week NCRT in patients with LARC.

\section{METHODS}

We performed a prospective, phase II study in patients with a histologically-proven, locally-advanced adenocarcinoma of the rectum. The study was approved by the relevant Institutional Review Board, and all patients gave written informed consent prior to any study procedure.

The initial work-up consisted of a complete history-taking and a physical examination, including digital rectal examination (DRE), rigid sigmoidoscopy, complete blood count, serum chemistry, carcinoembryonic antigen (CEA) test, chest radiography, and abdominopelvic computed tomography (CT). Disease-staging modalities included pelvic magnetic resonance imaging (MRI) or endorectal ultrasound for $\mathrm{T}$ and $\mathrm{N}$ stages, and positron emission tomography-CT (PET-CT) for metastasis in all patients. Full colonoscopy was performed and was alternated with flexible sigmoidoscopy up to the tumor when passage of the scope was impossible due to obstruction. Eligible patients had a histologically-verified adenocarcinoma within $12 \mathrm{~cm}$ from the anal verge, radiologic evidence of a T3 or T4 tumor, or node positive. Tumors were classified as lower $(<4 \mathrm{~cm}$ from the anal verge), middle $(4-8 \mathrm{~cm}$ from the anal verge), and upper ( $8-12 \mathrm{~cm}$ from the anal verge) rectal cancer according to their locations. Other inclusion criteria were an Eastern Cooperative Oncology Group score of 0-2, adequate function of major organs such as the heart, liver, kidneys and bone marrow, and signed informed consent. Exclusion criteria included other coexisting malignancies or a malignancy within the last 5 years prior to enrollment, severe concurrent medical or psychiatric disorders, prior RT to the pelvis and pregnancy or lactation.

RT and chemotherapy began simultaneously. RT was delivered to the whole pelvis by using a three-field approach at a dose of 45 Gy with daily doses of $1.8 \mathrm{~Gy}$ in 25 fractions, followed by a boost of $5.4 \mathrm{~Gy}$ in 3 fractions within 6 weeks, totaling $50.4 \mathrm{~Gy}$. Oral capecitabine at a dose of $825 \mathrm{mg} / \mathrm{m}^{2}$ twice daily with vitamin B6 for 6 weeks was administered concomitantly with RT. After completion of NCRT, consecutive additional 4-week chemotherapy was administered at the same dose. Radical surgery was performed 2-4 weeks following the completion of chemotherapy by expert colorectal surgeons. A low anterior resection, a coloanal anastomosis, or an abdominoperineal resection was performed on the principle of total mesorectal excision. En bloc tumor resection was performed when adjacent organ invasion was suspected, but a lateral pelvic lymph node dissection was not performed routinely. A low colorectal or total proctectomy with coloanal anastomoses was diverted with loop ileostomies at the surgeon's discretion.

The primary endpoint of the study was the pCR rate. The effect of NCRT was assessed by using the pretreatment radiologic TNM stage and the postoperative pathologic TNM stage. Patients were followed up after completion of 6-week NCRT. Toxicity was assessed according to the National Cancer Institute Common Toxicity Criteria, ver. 2.0 [11]. Tumor response was assessed 1 to 2 weeks after completion of chemotherapy by using physical examination, including DRE, CEA, flexible sigmoidoscopy, abdominopelvic CT, pelvic MRI, and PET-CT. Postoperative, pathological evaluations of the surgical specimens were performed by pathologists. The pathological response of the primary tumor was determined according to the College of American Pathologists (CAP) grade: CAP grade $0, \mathrm{pCR}$, indicating complete disappearance of all tumor cells; CAP grade 1, single cells or small groups of cancer cells (moderate response); CAP grade 2 , residual cancer outgrown by fibrosis (minimal response); CAP grade 3 , minimal or no tumor killed and extensive residual cancer (poor response).

The pCR rate in our previously published study with conventional NCRT was $20 \%$ [12]. We aimed to evaluate whether a pCR rate of $20 \%$ could be achieved by additional administration of capecitabine to conventional NCRT. A power analysis using G*Power 3 software (Duesseldorf, Germany) was performed to determine the appropriate sample size based on the desired power of 0.80 with an effect size of 0.25 , with the default value of $G^{*}$ Power 3 , and with the ratio of effect variance to the error variance, and with the level of significance set at 0.05 . Assuming that $>10 \%$ of patients would not be evaluated, the estimated sample size required was at least 44 patients. Statistics were descriptive, and all data were analyzed using the SPSS ver. 13 (SPSS Inc., Chicago, IL, USA).

\section{RESULTS}

Between January 2010 and September 2011, 44 patients were enrolled in the study. One patient expired 4 weeks after beginning NCRT due to massive bleeding of an underlying duodenal ulcer. Two patients discontinued chemotherapy 6 weeks after beginning NCRT, one because of grade 3 hand-foot syndrome (HFS) and the other because of grade 2 drug eruption. Therefore, 43 patients underwent surgery, and 41 patients completed the scheduled treatment (Table 1). Two patients with a tumor at the upper one-third of the rectum had multiple peritumoral and presacral lymph-node metastases with suspected extranodal manifestation to presacral 


\section{Coloproctology Kyung Ha Lee, et al.}

Table 1. Clinical characteristics $(n=44)$

\begin{tabular}{lc}
\hline Characteristic & Value \\
\hline Age (yr) & $65(35-86)$ \\
Sex & \\
Male & $30(68.2)$ \\
Female & $14(31.8)$ \\
ECOG performance status & \\
0 & $1(2.3)$ \\
1 & $43(97.7)$ \\
Tumor location (cm) & \\
$\leq 4$ & $16(36.4)$ \\
$4-8$ & $26(59.1)$ \\
$8-12$ & $2(4.5)$ \\
Radiologic study & \\
Computed tomography & $44(100)$ \\
Endorectal ultrasound & $11(25.0)$ \\
MRI & $41(93.2)$ \\
PET-CT & $42(95.5)$ \\
Clinical T stage & \\
T2 & $1(2.3)$ \\
T3 & $42(95.5)$ \\
T4 & $1(2.3)$ \\
No & \\
N1 & $6(13.9)$ \\
\hline Nacal N stage & $25(58.1)$ \\
\hline
\end{tabular}

Values are presented as median (range) or number (\%).

ECOG, Eastern Cooperative Oncology Group; MRI, magnetic resonance imaging; PET-CT, positron emission tomography-computed tomography.

vessels.

Surgical and pathologic characteristics are presented in Table 2. Nine cases of complications occurred immediately after surgery. Except for one patient who underwent a loop ileostomy due to a rectovaginal fistula, the other eight patients had minor complications of bleeding, surgical site infection, obstruction, or voiding difficulty, which did not need any surgical treatment. The pCR was noted in 9 patients (20.9\%). T down-staging and $\mathrm{N}$ downstaging were observed in 32 patients (74.4\%) and 33 patients (76.7\%), respectively (Table 3). One patient was diagnosed as being in stage IV after NCRT and underwent a synchronous hepatectomy with lower anterior resection. That patient was enrolled in the study as clinical stage T3N2M0 at first; however, a single liver metastasis was found on CT after NCRT. He discontinued capecitabine after 6 weeks due to grade 3 HFS. Toxicities are presented in Table 4 . Grade 3 to 5 toxicity was noted in 5 patients (11.4\%). Grade 3 HFS appeared in only 3 patients, and grade 1 or 2 HFS appeared in 19 patients and improved after discontinuing the drug for $1-2$ weeks or reducing the dosage to $50-75 \%$ of the initial value without any treatment.
Table 2. Surgical and pathologic characteristics $(n=43)$

\begin{tabular}{|c|c|}
\hline Characteristic & Value \\
\hline \multicolumn{2}{|l|}{ Type of surgery } \\
\hline Lower anterior resection & $30(69.8)$ \\
\hline Coloanal anastomosis & $9(20.9)$ \\
\hline Abdominoperineal resection & $4(9.3)$ \\
\hline \multicolumn{2}{|l|}{ TME grade } \\
\hline Incomplete & $0(0)$ \\
\hline Nearly complete & $1(2.3)$ \\
\hline Complete & $42(97.7)$ \\
\hline \multicolumn{2}{|l|}{ Postoperative complication } \\
\hline None & $34(79.1)$ \\
\hline Bleeding & $1(2.3)$ \\
\hline Surgical site infection & $4(9.3)$ \\
\hline Obstruction & $2(4.7)$ \\
\hline Voiding difficulty & $1(2.3)$ \\
\hline Rectovaginal fistula & $1(2.3)$ \\
\hline \multicolumn{2}{|l|}{ Histologic type } \\
\hline $\mathrm{pCR}$ & $9(20.9)$ \\
\hline Adenocarcinoma, WD & $2(4.7)$ \\
\hline Adenocarcinoma, MD & $30(69.8)$ \\
\hline Adenocarcinoma, PD & $0(0)$ \\
\hline Mucinous carcinoma & $2(4.7)$ \\
\hline \multicolumn{2}{|l|}{ Pathologic T stage } \\
\hline $\mathrm{pCR}$ & $9(20.9)$ \\
\hline TX, Tis, T1 & $6(13.9)$ \\
\hline $\mathrm{T} 2$ & $17(39.5)$ \\
\hline $\mathrm{T} 3, \mathrm{~T} 4$ & $11(25.6)$ \\
\hline \multicolumn{2}{|l|}{ Pathologic N stage } \\
\hline $\mathrm{pCR}$ & $9(20.9)$ \\
\hline NO & $22(51.2)$ \\
\hline N1 & $10(23.3)$ \\
\hline N2 & $2(4.6)$ \\
\hline \multicolumn{2}{|l|}{ Stage } \\
\hline pCR & $9(20.9)$ \\
\hline I & $16(37.2)$ \\
\hline$\|$ & $6(14.0)$ \\
\hline III & $11(25.6)$ \\
\hline IV & $1(2.3)$ \\
\hline \multicolumn{2}{|l|}{ Tumor regression grade } \\
\hline 0 & $9(20.9)$ \\
\hline 1 & $10(23.3)$ \\
\hline 2 & $18(41.9)$ \\
\hline 3 & $6(14.0)$ \\
\hline Metastatic lymph nodes & $0.7(0-9)$ \\
\hline Retrieved lymph nodes & $6(1-16)$ \\
\hline
\end{tabular}

TME, total mesorectal excision; pCR, pathologic complete response; WD, well differentiated; MD, moderately differentiated; PD, poorly differentiated. 
Table 3. Down-staging between preoperative clinical stages and postoperative pathological stages $(n=43)$

\begin{tabular}{lccccccc}
\hline $\begin{array}{l}\text { Preoperative } \\
\text { clinical stages }\end{array}$ & \multicolumn{7}{c}{ Postoperative pathological stages } \\
\cline { 2 - 8 } & pCR & TX, Tis, T1 & T2 & T3, T4 & N0 & N1 & N2 \\
\hline T2 & - & 1 & - & - & & & \\
T3 & 9 & 5 & 16 & 11 & & & \\
T4 & - & - & 1 & - & & & \\
N0 & 2 & & & & 4 & - & - \\
N1 & 1 & & & & 7 & 4 & - \\
N2 & 6 & & & & 11 & 6 & 2 \\
\hline
\end{tabular}

$\mathrm{pCR}$, pathologic complete response.

\section{DISCUSSION}

Although NCRT has been used as standard treatment modality in patients with LARC, distant metastasis is still being reported in up to $30 \%$ of the patients, and the survival benefit of NCRT has not been demonstrated. These facts can probably be attributed to the existence of microscopic lesions prior to treatment and to a varied biochemical response of tumors to RT. A study reported a burst of rapid repopulation of remnant tumor cells after a lag period following RT [13]. Numerous studies have tried to find ways to decrease distant metastasis and ultimately to improve survival in patients who are candidates for NCRT. The final pathologic features have been demonstrated to be the most significant prognostic factors in rectal cancer [5]. Therefore, we assumed that survival would improve in patients who received NCRT if a large pCR were achieved. The pCR has been reported to be associated with excellent long-term survival [6].

In order to increase the $\mathrm{pCR}$ rate, diverse regimens of chemoradiotherapy have been tried. There were limitations to increasing the dosage of radiation because of radiation toxicity. However, a chemotherapeutic regimen can be adjusted in a safer and more variable manner. Adding oxaliplatin, irinotecan, or several biologic agents, including cetuximab, bevacizumab, erlotinib or panitumumab, to conventional 5-FU-based chemotherapy has been tried. Although those regimens were demonstrated to be effective and tolerable, none of them obtained superior results when compared to conventional 5-FU-based chemotherapy in terms of the pCR rate or the survival [14-17]. Induction chemotherapy is reported to show no advantage in efficacy and to have rather more toxicity [18]. Contrary to adding new drugs or induction chemotherapy, increasing the duration of chemotherapy was considered so as not to increase the toxicity. If increasing the duration of chemotherapy improved the effect of NCRT, it might finally be a reasonable way to increase the $\mathrm{pCR}$ and the survival rate.

Few studies have addressed the effect of additional chemotherapy during resting periods. Habr-Gama et al. [10] reported that the clinical complete remission rate was increased to $65 \%$ by increasing the duration of 5-FU/leucovorin from 3 cycles to 6 cycles
Table 4. Acute toxicity during preoperative chemoradiotherapy $(\mathrm{n}=$ 44)

\begin{tabular}{lccccc}
\hline \multirow{2}{*}{ Toxicity } & \multicolumn{5}{c}{ Patient } \\
\cline { 2 - 6 } & Grade 1 & Grade 2 & Grade 3 & Grade 4 & Grade 5 \\
\hline None (43.2\%) & 19 & & & & \\
Hand-foot syndrome (50.0\%) & 10 & 9 & 3 & - & - \\
Enteritis (2.3\%) & - & - & 1 & - & - \\
Dermatitis (2.3\%) & - & 1 & - & - & - \\
Drug eruption (2.3\%) & - & 1 & - & - & - \\
Ulcer (2.3\%) & - & - & - & - & 1 \\
\hline
\end{tabular}

as NCRT. They presumed that the high rate of complete remission could be a result of the extended exposure of radiated tumor cells to chemotherapy. Their study inspired our study by suggesting that increasing the duration of chemotherapy could increase the $\mathrm{pCR}$ rate.

The Fédération Francophone de Cancérologie Digestive 9203 and European Organisation for Research and Treatment of Cancer Radiotherapy Group Trial 2,2921 reported that addition of chemotherapy to preoperative RT improved local control significantly; however, no survival benefit was demonstrated $[3,8]$. The role of chemotherapy concomitant to RT in a preoperative setting was known to be that of a radiosensitizer used to enhance the therapeutic efficacy of radiation [9]. Fluorouracil is the most wellknown radiosensitizing drug, and hjas been suggested as playing a key role for dysregulation of S-phase checkpoints. In the presence of 5-FU, radiosensitivity was increased in relatively radioresistant cells that progress inappropriately into the Sphase [19]. The group with addition of 5-FU to preoperative RT is reported to show more inactivation of the proliferating population of the tumor with respect to mitotic count, Ki-67, and proliferating cell nuclear antigen immunostaining when compared with the radiation-only group [20]. In the present study, additional chemotherapy following conventional NCRT was tried, expecting not only a radiosensitizer effect but also its own tumoricidal effect, thus increasing the pCR rate. Our study used oral capecitabine instead of intravenous 5-FU, and the regimen was assumed to have advantages in terms of compliance, convenience and economy.

Capecitabine (N4-pentyloxycarbonyl-5'-deoxy-5-fluorocytidine, Xeloda, Hoffmann-La Roche Ltd., Basel, Switzerland) is an oral prodrug that is metabolized to 5-FU in vivo and mimics the pharmacokinetics of continuous 5-FU infusion. Two phase-III trials, the large National Surgical Adjuvant Breast and Bowel Project R-04 Intergroup study [21] and a German trial [22], have confirmed that capecitabine was not inferior to 5-FU for NCRT in rectal cancer, and one retrospective analysis found preoperative capecitabine plus RT to have more favorable results and a higher down-staging rate than infusional 5-FU plus RT [23]. Unlike 5-FU, which is administered continuously with a need for a vascular access port, the low toxicity and the high compliance due to 
capecatibine's oral administration makes the NCRT more tolerable and patient-friendly, with the expectation of replacing continuous infusional 5-FU. Increasing the duration of chemotherapy was acceptable because of these advantages. If additional capecitabine increased the $\mathrm{PCR}$ rate without significant increase of toxicity, it might be expected to contribute to improved survival.

In the present study, most side effects were tolerable, and grade 3 to 5 toxicity rates did not increase compared to those of conventional NCRT (>15\%) [24]. HFS was known to be one of the most common adverse effects of capecitabine, with an incidence rate of $45-68 \%$ [25]. In the present study, HFS appeared in 50\% of the patients, similar to other reports. Although hematologic toxicity and diarrhea are known to be common adverse effects and have been reported in up to $50 \%$ and $25 \%$ of the patients [22], respectively, hematologic toxicity was not found, and diarrhea was found in one patient in the present study. Most toxicities did not require any treatment and improved by themselves. Therefore, an increased duration of chemotherapy did not increase toxicity.

Despite the advantages of additional chemotherapy predicted above, the present study obtained a pCR rate of $20.9 \%$, which was similar to the pCR rates obtained after conventional NCRT in our institute and others [7]. Therefore, additional chemotherapy did not increase the PCR rate compared with conventional NCRT or with other various experimental regimens with antitumor drugs or target agents. As a limitation of the present study, the dosage of additional capecitabine without RT was 1,650 mg per day as a radiosensitizer, which was less than 2,500 $\mathrm{mg}$ per adjuvant chemotherapy. This may be considered as one possible reason for this not being a superior result.

The long-term oncologic benefit of additional chemotherapy could not be estimated in the present study. The pCR rate is the most commonly used primary endpoint in many studies regarding NCRT in rectal cancers because its assessment requires a short period of study. However, additional chemotherapy may improve long-term survival by eliminating micrometastatic lesions without significantly increasing the pCR rate [26]. Moreover, Bonnetain et al. [27] insisted that the pCR did not qualify as a surrogate for overall or progression-free survival [27].

Distant metastasis after acquiring a pCR is reported to be $8.7 \%$ [28]. Distant micrometastases could remain despite sterilization of tumor cells around the tumor's origin in the pelvis. Neoadjuvant chemotherapy has theoretical advantages over the potential to eradicate early distant micrometastases, which may already exist at initial diagnosis or may grow during preoperative treatment [29]. Although the resting period is essential when preparing for surgery, it could be considered as a delay of definite surgery and as a vacancy of treatment. During this period, additional oral chemotherapy could reduce the chance of potential disease progression. Therefore, a further comparison study with enough longterm follow-up is required to assess the direct effect of additional chemotherapy on survival.

In conclusion, this study showed that additional 4-week chemo- therapy with capecitabine during the resting periods after 6-week NCRT was safe, however, it was no more effective than conventional NCRT.

\section{CONFLICT OF INTEREST}

No potential conflict of interest relevant to this article was reported.

\section{REFERENCES}

1. Sauer R, Becker H, Hohenberger W, Rodel C, Wittekind C, Fietkau $\mathrm{R}$, et al. Preoperative versus postoperative chemoradiotherapy for rectal cancer. N Engl J Med 2004;351:1731-40.

2. Bosset JF, Collette L, Calais G, Mineur L, Maingon P, RadosevicJelic L, et al. Chemotherapy with preoperative radiotherapy in rectal cancer. N Engl J Med 2006;355:1114-23.

3. Gerard JP, Conroy T, Bonnetain F, Bouche O, Chapet O, ClosonDejardin MT, et al. Preoperative radiotherapy with or without concurrent fluorouracil and leucovorin in T3-4 rectal cancers: results of FFCD 9203. J Clin Oncol 2006;24:4620-5.

4. Valentini V, van Stiphout RG, Lammering G, Gambacorta MA, Barba MC, Bebenek M, et al. Nomograms for predicting local recurrence, distant metastases, and overall survival for patients with locally advanced rectal cancer on the basis of European randomized clinical trials. J Clin Oncol 2011;29:3163-72.

5. Huebner M, Wolff BG, Smyrk TC, Aakre J, Larson DW. Partial pathologic response and nodal status as most significant prognostic factors for advanced rectal cancer treated with preoperative chemoradiotherapy. World J Surg 2012;36:675-83.

6. Zorcolo L, Rosman AS, Restivo A, Pisano M, Nigri GR, Fancellu A, et al. Complete pathologic response after combined modality treatment for rectal cancer and long-term survival: a meta-analysis. Ann Surg Oncol 2012;19:2822-32.

7. Roh MS, Colangelo LH, O'Connell MJ, Yothers G, Deutsch M, Allegra CJ, et al. Preoperative multimodality therapy improves disease-free survival in patients with carcinoma of the rectum: NSABP R-03. J Clin Oncol 2009;27:5124-30.

8. Bosset JF, Calais G, Mineur L, Maingon P, Radosevic-Jelic L, Daban A, et al. Enhanced tumorocidal effect of chemotherapy with preoperative radiotherapy for rectal cancer: preliminary results: EORTC 22921. J Clin Oncol 2005;23:5620-7.

9. Byfield JE, Chan PYM, Seagren S. Radiosensitization by 5-fluorouracil (5-FU): molecular and clinical scheduling implications. Proc Am Assoc Cancer Res 1977;18:74.

10. Habr-Gama A, Perez RO, Sabbaga J, Nadalin W, Sao Juliao GP, Gama-Rodrigues J. Increasing the rates of complete response to neoadjuvant chemoradiotherapy for distal rectal cancer: results of a prospective study using additional chemotherapy during the resting period. Dis Colon Rectum 2009;52:1927-34.

11. Trotti A, Byhardt R, Stetz J, Gwede C, Corn B, Fu K, et al. Common toxicity criteria: version 2.0. an improved reference for grad- 
ing the acute effects of cancer treatment: impact on radiotherapy. Int J Radiat Oncol Biol Phys 2000;47:13-47.

12. Kim JY. Oncologic result as according to tumor regression grade after neoadjuvant chemoradiation therapy in locally advanced rectal cancer. Journal of the Korean Society of Coloproctology 2008; 24:422-32.

13. Withers HR, Taylor JM, Maciejewski B. The hazard of accelerated tumor clonogen repopulation during radiotherapy. Acta Oncol 1988;27:131-46.

14. Aschele C, Cionini L, Lonardi S, Pinto C, Cordio S, Rosati G, et al. Primary tumor response to preoperative chemoradiation with or without oxaliplatin in locally advanced rectal cancer: pathologic results of the STAR-01 randomized phase III trial. J Clin Oncol 2011;29:2773-80.

15. Dewdney A, Cunningham D, Tabernero J, Capdevila J, Glimelius B, Cervantes A, et al. Multicenter randomized phase II clinical trial comparing neoadjuvant oxaliplatin, capecitabine, and preoperative radiotherapy with or without cetuximab followed by total mesorectal excision in patients with high-risk rectal cancer (EXPERT-C). J Clin Oncol 2012;30:1620-7.

16. Blaszkowsky LS, Hong TS, Zhu AX, Kwak LE, Mamon HJ, Shellito PC, et al. A phase I/II study of bevacizumab (beva), erlotinib (erl), and 5-fluorouracil (5-FU) with concurrent external beam radiation therapy (RT) in locally advanced rectal cancer (LARC) [abstract]. J Clin Oncol 2009;27(15 Suppl);4106.

17. Pinto C, Di Fabio F, Maiello E, Pini S, Latiano T, Aschele C, et al. Phase II study of panitumumab, oxaliplatin, 5-fluorouracil, and concurrent radiotherapy as preoperative treatment in high-risk locally advanced rectal cancer patients (StarPan/STAR-02 Study). Ann Oncol 2011;22:2424-30.

18. Fernandez-Martos C, Pericay C, Salud A, Massuti B, Alonso V, Safont MJ, et al. Three-year outcomes of GCR-3: a phase II randomized trial comparing conventional preoperative chemoradiation (CRT) followed by surgery and postoperative adjuvant chemotherapy (CT) with induction CT followed by CRT and surgery in locally advanced rectal cancer [abstract]. J Clin Oncol 2011;29 (15 Suppl):3552.

19. Lawrence TS, Blackstock AW, McGinn C. The mechanism of action of radiosensitization of conventional chemotherapeutic agents. Semin Radiat Oncol 2003;13:13-21.

20. Willett CG, Hagan M, Daley W, Warland G, Shellito PC, Compton
CC. Changes in tumor proliferation of rectal cancer induced by preoperative 5-fluorouracil and irradiation. Dis Colon Rectum 1998;41:62-7.

21. Roh S, Yothers GA, O'Connell MJ, Beart RW, Pitot HC, Shields $\mathrm{AF}$, et al. The impact of capecitabine and oxaliplatin in the preoperative multimodality treatment in patients with carcinoma of the rectum: NSABP R-04 [abstract]. J Clin Oncol 2011;29(15 Suppl): 3503.

22. Hofheinz RD, Wenz F, Post S, Matzdorff A, Laechelt S, Hartmann JT, et al. Chemoradiotherapy with capecitabine versus fluorouracil for locally advanced rectal cancer: a randomised, multicentre, non-inferiority, phase 3 trial. Lancet Oncol 2012;13:579-88.

23. Saif MW, Hashmi S, Zelterman D, Almhanna K, Kim R. Capecitabinevs continuous infusion 5-FU in neoadjuvant treatment of rectal cancer: a retrospective review. Int J Colorectal Dis 2008;23: 139-45.

24. Dunst J, Debus J, Rudat V, Wulf J, Budach W, Hoelscher T, et al. Neoadjuvant capecitabine combined with standard radiotherapy in patients with locally advanced rectal cancer: mature results of a phase II trial. Strahlenther Onkol 2008;184:450-6.

25. Abushullaih S, Saad ED, Munsell M, Hoff PM. Incidence and severity of hand-foot syndrome in colorectal cancer patients treated with capecitabine: a single-institution experience. Cancer Invest 2002;20:3-10.

26. Berardi R, Maccaroni E, Onofri A, Giampieri R, Bittoni A, Pistelli $\mathrm{M}$, et al. Multidisciplinary treatment of locally advanced rectal cancer: a literature review. Part 1. Expert Opin Pharmacother 2009; 10:2245-58.

27. Bonnetain F, Bosset JF, Gerard JP, Calais G, Conroy T, Mineur L, et al. What is the clinical benefit of preoperative chemoradiotherapy with 5FU/leucovorin for T3-4 rectal cancer in a pooled analysis of EORTC 22921 and FFCD 9203 trials: surrogacy in question? Eur J Cancer 2012;48:1781-90.

28. Martin ST, Heneghan HM, Winter DC. Systematic review and meta-analysis of outcomes following pathological complete response to neoadjuvant chemoradiotherapy for rectal cancer. Br J Surg 2012;99:918-28.

29. Glynne-Jones R, Grainger J, Harrison M, Ostler P, Makris A. Neoadjuvant chemotherapy prior to preoperative chemoradiation or radiation in rectal cancer: should we be more cautious? Br J Cancer 2006;94:363-71. 